\title{
Analysis of in situ Nanodiamonds in Organic Matter from Primitive Meteorites with Electron Energy-Loss Spectroscopy and Energy Dispersive X-ray Spectroscopy
}

\author{
Bradley T. De Gregorio ${ }^{1 *}$, Conel M. O’D. Alexander ${ }^{2}$, and Rhonda M. Stroud ${ }^{1}$ \\ 1. Materials Science and Technology Division, U.S. Naval Research Laboratory, Washington, DC USA \\ 2. Department of Terrestrial Magnetism, Carnegie Institution of Washington, Washington, DC USA \\ * Corresponding author: bradley.degregorio@ nrl.navy.mil
}

Nanodiamonds are present in primitive meteorites at concentrations of up to a few thousand ppm (by mass) $[1,2]$. However, the origin of these nanodiamonds is controversial. Bulk measurements of noble gases (e.g. Ar, $\mathrm{Xe}, \mathrm{Kr}$ ) and trace elements indicate that they contain nucleosynthetic products of supernovae, yet major element $(\mathrm{C}$ and $\mathrm{N}$ ) isotopic compositions lie within solar system values. Thus, multiple formation environments are possible, including within the early solar nebula, the interstellar medium, or from other stars [3,4]. This uncertainty has been maintained because the isotopic composition of individual $\sim 2 \mathrm{~nm}$ nanodiamonds cannot be reliably measured by current methods, although some progress is being made using atom-probe tomography $[5,6]$.

One way to circumvent the need for single nanodiamond isotopic analyses may be to locate nanodiamonds in situ within fine-grained meteorite matrix [7]. This would provide petrographic context for nanodiamond formation if they are preferentially associated with material of a specific cosmochemical provenance, i.e., organic matter with ${ }^{15} \mathrm{~N}$ enrichment. In this study, we use scanningtransmission electron microscopy (STEM) to locate nanodiamonds in insoluble organic matter (IOM) isolates from three primitive meteorites-Murchison, EET92042, and DOM08006. IOM was isolated from their host meteorites through a multi-step, room temperature $\mathrm{HCl} / \mathrm{CsF}-\mathrm{HF}$ dissolution process [8]. Isolate particles were sulfur-embedded, ultramicrotomed, and $90 \mathrm{~nm}$ sections were placed on lacey $\mathrm{C}$ TEM grids. Sample analysis was performed in a Nion UltraSTEM 200, operated at $60 \mathrm{keV}$ with a probe current of $~ 120$ pA. This microscope includes a Gatan Enfinia electron energy-loss spectrometer (EELS) and a 0.7 sr windowless, Bruker energy dispersive X-ray spectrometer (EDS).

To locate the nanodiamonds, we used variations on methods previously-used for spatially-resolving $\mathrm{sp}^{2}$ and $\mathrm{sp}^{3}$ carbons, including diamond [8]. The band structure of diamond is distinct from that of the aromatic amorphous $\mathrm{C}$ typical of IOM, and thus spectral features of both the C core-loss and low-loss regions of the EELS spectrum can be used [Figure 1]. We settled on a simple metric for diamond detection, either the difference between $1 \mathrm{eV}$ bands centered at $285.0 \mathrm{eV}$ (where $\mathrm{C}=\mathrm{C}$ strongly absorbs and is absent in diamond) and $289.3 \mathrm{eV}$ (at the leading edge of the diamond $\sigma^{*}$ peak) for core-loss spectra [Figure 1B], or the difference between $1 \mathrm{eV}$ bands centered at the IOM bulk plasmon at $23 \mathrm{eV}$ and the diamond bulk plasmon at $34 \mathrm{eV}$ for low-loss spectra [Figure 1D]. Both methods are essentially measuring the average slope of the spectra between the two selected bands, and produce similar quality maps of diamond in the IOM, although the plasmon approach can map a larger area at the same spatial resolution at a fraction of the time, due to the higher intensity of the EELS spectrum at those energies.

Our EELS maps indicate that nanodiamonds are distributed heterogeneously throughout the IOM isolates. The maps show good correspondence with intensity variations in MAADF images, but not HAADF images, due to the minor density difference between diamond and IOM. The greatest concentrations are found in "fluffy" textured IOM, and are notably absent in non-porous, compact 
organic matter and nanoglobules. This may be expected since much of this type of IOM likely formed in the parent asteroids, and the nanodiamonds most likely formed prior to asteroid formation. We also found some clusters of nanodiamonds that appear to be associated with $\mathrm{N}$ enrichments of up to $40 \%$ [Figure 2]. Since this level of $\mathrm{N}$ cannot be incorporated into the nanodiamonds themselves, it indicates that these clusters are associated with N-rich IOM, and likely have a common cosmochemical origin that could be further elucidated by $\mathrm{N}$ isotope measurements.

\section{References:}

[1] R Lewis et al., Nature 328 (1987), p. 160.

[2] G Huss et al., Geochimica et Cosmochimica Acta 67 (2003), p. 4823.

[3] Z Dai et al., Nature 418 (2002), p. 157.

[4] R Stroud et al., Astrophysical Journal Letters 738 (2011), L27.

[5] J Lewis et al., Ultramicroscopy 159 (2015), p. 248.

[6] J Lewis et al., Microscopy and Microanalysis 23 S1 (2017), p. 678.

[7] L Garvie, Lunar and Planetary Science Conference XLI (2010), abstract 1388.

[8] C Alexander et al., Geochimica et Cosmochimica Acta 221 (2018), p. 406.

[9] M Bosman et al., Carbon 47 (2009), p. 94.
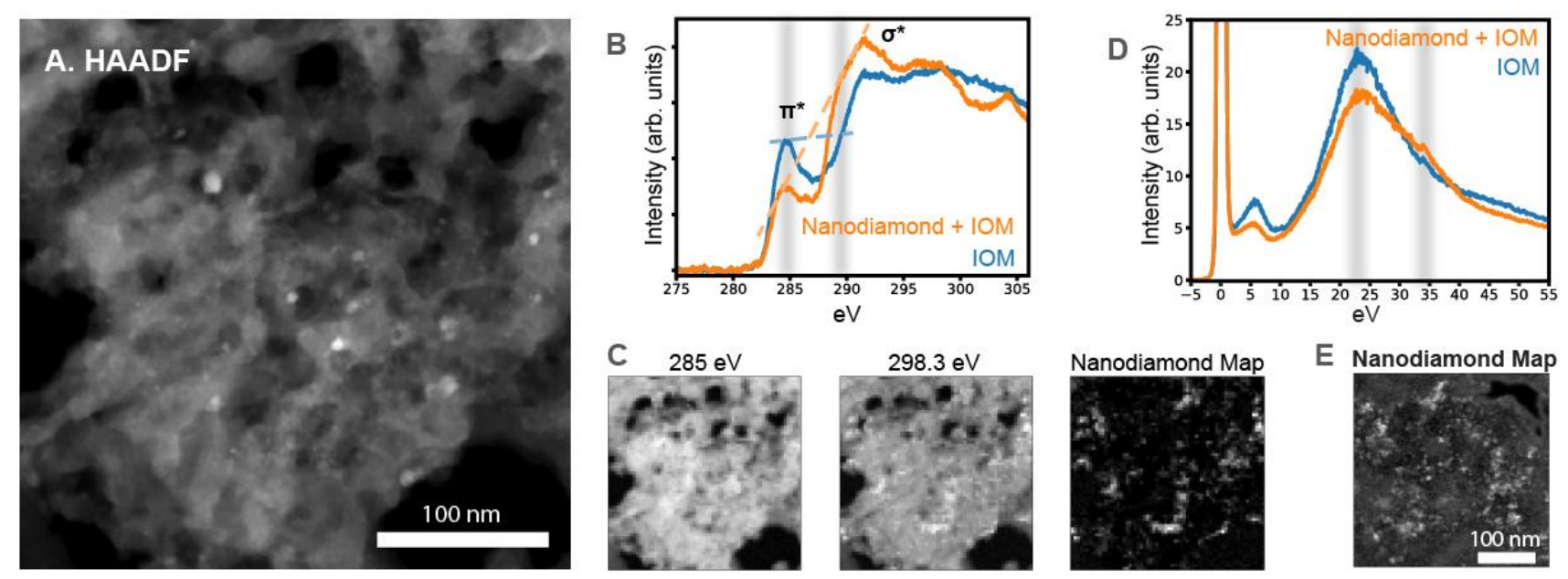

Figure 1. (A) STEM-HAADF image of Murchison IOM. Bright spots are chromites and other high-Z grains, while nanodiamonds are invisible. (B) Core-loss C-EELS spectra of IOM and nanodiamond. (C) EELS spectrum image slices at energies shown in (B), along with a map of nanodiamond distribution. (D) Low-loss EELS spectra of IOM and nanodiamond. (E) Low-loss map of nanodiamond distribution.
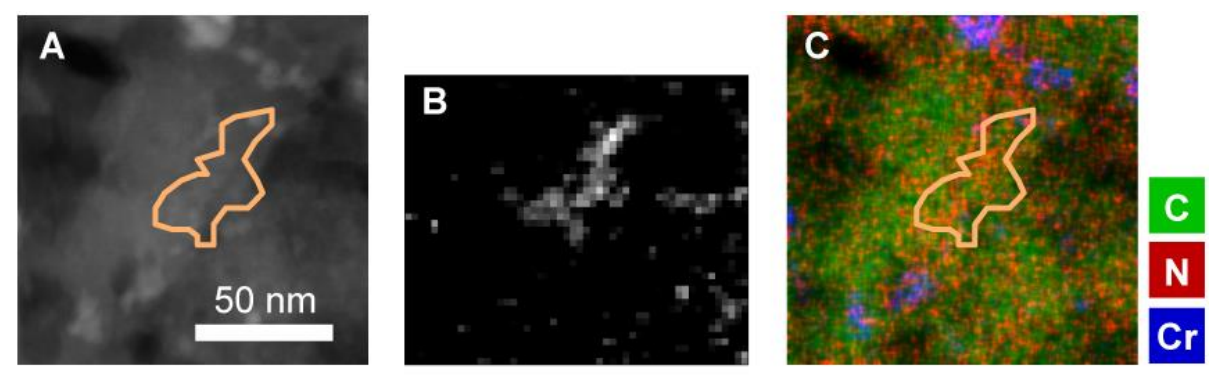

Figure 2. (A) STEM-MAADF image of an invisible cluster of nanodiamonds in Murchison IOM. (B) Corresponding diamond map. (C) Corresponding EDS map indicating a $\sim 40 \%$ enrichment in N. 